Pérez, S.; Rodríguez, A.; Sánchez, A.; De Mena, J.M.; Fuentes, J.M.; Castaño, R. y Martín, N. (2019). Efecto de los juegos reducidos sobre jugadoras de fútbol / Effect of Small-Sided Games on Football Players. Revista Internacional de Medicina y Ciencias de la Actividad Física y el Deporte vol. 19 (74) pp. 371-386 Http://cdeporte.rediris.es/revista/revista74/artefecto1031.htm DOI: http://doi.org/10.15366/rimcafd2019.74.012

\title{
ORIGINAL
}

\section{EFECTO DE LOS JUEGOS REDUCIDOS SOBRE JUGADORAS DE FÚTBOL}

\section{EFFECT OF SMALL-SIDED GAMES ON FOOTBALL PLAYERS}

\author{
Pérez, S.. ; Rodríguez, A. 1; Sánchez, A. ${ }^{1}$; De Mena, J.M.'; Fuentes, J.M.. \\ Castaño, R. ${ }^{1}$ y Martín, N. ${ }^{3}$ \\ 1 Doctor en Ciencia de la Educación. Profesor del Grado de Ciencias de la Actividad Física y el \\ Deporte. Universidad Pontificia de Salamanca (España) sperezmu@upsa.es, \\ arodriguezca@upsa.es, asanchezmu01@upsa.es, jmenara@upsa.es, rcastanoca@upsa.es \\ 2 Doctor en Psicología. Profesor del Grado de Ciencias de la Actividad Física y el Deporte. \\ Universidad Pontificia de Salamanca. España. jmfuentesbl@upsa.es. \\ 3 Graduada en Educación Primara: Mención Educación Física. Universidad Pontificia de \\ Salamanca. España. noefrancos@hotmail.com.
}

\section{AGRADECIMIENTOS}

Los autores agradecen a los jóvenes y padres la predisposición mostrada para el desarrollo de este estudio. También, la incondicional colaboración del club Fútbol Femenino Salamanca, materializada en la ayuda de los técnicos y responsables de las categorías inferiores.

Código UNESCO: 5899 Educación Física y Deportiva / Physical education and sport.

Clasificación del Consejo de Europa: 17. Otras (iniciación deportiva) / Other (sport initiation).

Recibido 26 de julio de 2017 Received July 26, 2017

Aceptado 21 de noviembre de 2017 Accepted November 21, 2017

\section{RESUMEN}

El objetivo ha sido analizar cómo influyen los juegos reducidos o SSGs sobre la condición física, técnica y los cambios de dirección (CODA) en jugadoras alevines de fútbol femenino. Participan 12 jugadoras de fútbol femenino. Realizaron 14 sesiones de entrenamiento basado en juegos reducidos de fútbol. La muestra presenta valores normales en la prueba de Saphiro-Wilk, además, en la prueba de $\mathrm{t}$ de Student muestra que existen diferencias estadísticamente muy significativas $(p \leq 0,00)$ en todas las variables físicas analizadas, menos en el caso de la fuerza explosiva. En las variables de técnica 
nos muestra que existen diferencias significativas en todas las variables analizadas $(p \leq 0,00)$. Por último existen correlaciones positivas y estadísticamente significativas entre las distintas variables analizadas. De esta forma podemos considerar los juegos reducidos son un método adecuado y muy ventajoso para el entrenamiento en las etapas iniciales o de formación en jugadoras de fútbol.

PALABRAS CLAVE: Fútbol base; juegos reducidos; preparación física; Técnica; Mujer.

\section{ABSTRACT}

The objective has been to analyze how small-sided games or SSGs affect physical, technical and changes of direction (CODA) in U12 female soccer players. 12 female soccer players participate. They conducted 14 training sessions based on reduced soccer games. The sample has normal values in the Saphiro-Wilk test, and in the Student's t test it shows that there are statistically very significant differences $(p \leq 0,00)$ in all the analyzed physical variables, less in the case of the explosive force. In the technique variables we show that there are significant differences in all variables analyzed $(p \leq 0,00)$. Finally, there are positive and statistically significant correlations between the different variables analyzed. In this way we can consider reduced games are a suitable and very advantageous method for training in the initial stages or training in soccer players.

KEYWORDS: soccer learning; Small-sided games; physical-fitness; technique; Women.

\section{INTRODUCCIÓN}

El fútbol nació como un juego en distintos ámbitos y lugares. Su evolución a lo largo de los siglos lo han convertido hoy en día en el "deporte rey" (Vitoria, 2005). Por supuesto, en España es el deporte más practicado, destacando sobre los demás (Pérez, Sánchez \& Urchaga, 2015). Esto es aplicable al fútbol masculino, pero no es así al fútbol femenino que no tiene la misma repercusión. Los datos muestran que el número de licencias de jugadoras que es bastante inferior a lo de los hombres, 29.807 frente a las 684.320, respectivamente (RFEF, 2015). El fútbol es el deporte rey por excelencia, pero no es así para las mujeres que lo practican.

En los últimos años han aumentado los estudios sobre el fútbol rendimiento, dejando de lado a los jóvenes jugadores (González-Villoria, GarcíaLópez \& Contreras-Jordán, 2015). Sin embargo en el caso de la iniciación y formación son necesarios más investigaciones en las distintas categorías (González-Villoría, et al., 2015). Tampoco hay muchos estudios que se centren en estudiar a las mujeres que juegan al fútbol. Existen estudios que se centran en la percepción subjetiva del esfuerzo de jugadoras inactivas (Martínez, et al., 2015), en la cuantificación de los desplazamientos en fútbol siete infantil 
(Barbero-Álvarez, Barbero-Álvarez, Gómez \& Castagna, 2009), en la flexibilidad en juveniles de fútbol (Del Río, Flores, Bautista, Barajas, Tamara \& Gómez, 2015) y fútbol - sala (Ayala, De Baranda, Cejudo \& De Ste, 2010), la cohesión en el juego en cadetes (González, Sánchez, Amado, Pulido, López, \& Leo, 2013), y el más específico de Nevado-Garrosa y Suárez-Arrones (2015) sobre jugadoras sub 13 aunque miden exclusivamente los efectos físicos.

Los juegos reducidos o small-sided games (SSGs) (Rampinini et al., 2007) se encuadran dentro de los modelos modernos de enseñanza, tienen en cuenta su especificad (Dellal, Drust \& Lago-Penas, 2012) y la lógica interna (Hernández, 1994), escapando de los modelos tradicionales (Robles, Giménez \& Abad, 2011). Los juegos reducidos (JR) son situaciones motrices lúdico-deportivas (Parlebas, 2001) en las que se incluyen la mayor parte de los factores que intervienen en el juego real de una manera adaptable (Wein, 1995). Los SSGs son juegos que simulan el deporte total o parcialmente, y son un método adecuado para la mejora del rendimiento de los jugadores (Katis \& Kellis, 2009), por ello estaríamos hablando del mismo término. El espacio y las reglas se adaptan al número menor de jugadores (Little, 2009). Estas adaptaciones son suficientes para reproducir las situaciones que se producen en los partidos (Owen, Twist \& Ford, 2004), sirven para entrenar lo imprevisible y las demandas del fútbol (Cristian, Riller, René, Denise, Milton \& Alcides, 2015; Nevado-Garrosa \& Suárez-Arrones, 2015), además, son adecuados y muy populares con independencia de la edad y el nivel de los jugadores (Hill-Haas, Dawson, Impellizzeri \& Coutts, 2011), siendo especialmente necesarios en las etapas formación (Casamichana, San Román, Calleja \& Castellano, 2015). Estamos, por tanto, ante un tipo de tareas que recogen la complejidad y especificad del fútbol, donde los jugadores pueden mejorar todos los elementos implicados en el rendimiento (Dellal, Hill-Haas, Lago-Peñas \& Chamari, 2011), esto provoca que sean un medio de entrenamiento habitual en todos los niveles (Owen, Wong, Paul \& Dellal, 2012).

Existen variedad de estudios centrados en los SSGs que analizan la cuantificación la carga interna y la condición física (Casamichana, Castellano, González-Morán, García-Cueto \& García-López, 2011; Dellal, et al., 2011; HillHaas, et al, 2011; Kelly \& Drust 2009; Little \& Williams, 2006; Martone, et. al., 2017; Owen, et al., 2012), los aspectos técnicos (Pérez, Sánchez, Sánchez \& Yagüe, 2012; Sánchez, Yagüe, Fernández \& Petisco, 2014; Soto \& Pérez, 2014a), aspectos tácticos como los tiempo de juego, posesión y duración de las jugadas (Soto \& Pérez, 2014b) y otros sobre la manipulación de elementos propios del juego como: el número de jugadores, el espacio, presencia de porteros, demarcaciones, distribución de equipos y las reglas (Abrantes, Nunes, Maças, Leite \& Sampaio, 2012; Castellano, Casamichana \& Dellal, 2013; Fradua, Zubillaga, Caro, Fernández-García, Ruiz-Ruiz \& Tenga, 2013; Gonçalves, Figueira, Maças \& Sampaio, 2014).

Antes de terminar, los cambios de dirección (CODA) son uno de los parámetros determinantes del rendimiento en jugadores de fútbol (Bangsbo, Mohr \& Krustrup, 2006; Pardeiro \& Yanci, 2017; Santiago, Granados, Quintela \& Yanci, 2015) y en otros deportes (Yanci, Castillo, Vizcay, Pitillas \& Iturricastillo, 2016), aunque no de forma exclusiva, ya que son necesarios también potencia 
aeróbica, fuerza y la velocidad (Yanci, García, Castillo \& Rivero, 2014). Si bien las investigaciones en este campo se centran en etapas de rendimiento y población masculina. En este sentido hay pocos trabajos sobre el efecto de los juegos reducidos (SSGs) sobre los CODA, así por ejemplo, esta el trabajo realizado por Yanci, Reina, Los Arcos y Cámara (2013), que estudian el efecto de la interferencia contextual el la mejora de los CODA, en una muestra de menor edad y comparando niños y niñas de primer curso de primaria.

Por último, hay estudios en jugadores jóvenes masculinos, como se ha indicado, sin embargo en el caso de las mujeres es escaso este tipo de estudios, tampoco existen estudios que analicen los cambios de dirección (CODA) en estas etapas. Por todo ello el objetivo del estudio es analizar cómo influyen los juegos reducidos o SSGs sobre la condición física, técnica y los cambios de dirección (CODA) en jugadoras alevines de fútbol.

\section{MATERIAL Y MÉTODOS}

\subsection{METODOLOGÍA}

La investigación que se realiza es de tipo cuantitativa experimental a través de un estudio de campo con un pre-test, intervención y un pos-test final, para comprobar el efecto de la intervención sobre la condición física. Cook y Reichardt (1986) y Pita y Pértegas (2002) indican que es una metodología de investigación cuantitativa, ya que se recogen datos observables, medibles, cuantificables y se ofrecen unos resultados numéricos.

\subsection{MUESTRA}

La muestra la forman 12 jugadoras alevines con alto nivel de pericia (tabla 1), con edad media de 11,50 ( $\pm 0,511$ años), con un peso de 43,25 ( $\pm 8,001 \mathrm{~kg}$.) y una altura media de $150,5 \mathrm{~cm}$. $( \pm 10,475)$. Todas ellos pertenecen al club de Fútbol Femenino Salamanca que compite en la categoría alevín jugando contra el resto de equipos que están formados por niños. Es el único equipo que está compuesto exclusivamente por jugadoras, por lo tanto la muestra representa el $85,71 \%$ de la población objeto de estudio.

Tabla 1. Principales características de la muestra participante en el estudio

\begin{tabular}{ccccccc}
\hline $\mathrm{N}$ & Edad $(\bar{x})$ & Edad $(S \bar{x})$ & Peso $(\bar{x})$ & Peso $(S \bar{x})$ & Altura $(\bar{x})$ & Altura $(S \bar{x})$ \\
\hline 12 & 11,50 años & 0,511 & $43,25 \mathrm{Kg}$ & 8,001 & $150,50 \mathrm{~cm}$ & 10,475 \\
\hline
\end{tabular}

\subsection{MATERIAL}

La parte experimental se ha desarrollado en las instalaciones del club en todas las áreas: material de campo, campo de entrenamiento y vestuarios. Todas las mediciones se realizaron en el campo de entrenamiento habitual.

Los test físicos - técnicos realizados fueron los siguientes: 
- Salto a pies juntos: Las jugadoras se sitúan desde la posición inicial, sin carrera o movimientos previos tenían que saltar hacía delante para alcanzar la mayor distancia posible, cayendo con los dos pies en el mismo sitio (Amaya®, precisión $2 \mathrm{~mm}$ ).

- Course Navette (resistencia aeróbica): Las jugadoras recorren de forma ininterrumpida la distancia de $20 \mathrm{~m}$. pisando la línea señalada, siguiendo las señales acústicas amplificadas con altavoz (altavoces Sony ENG203®) desde un ordenador (Acer ${ }^{\circledR}$ TravleMate 5720). Se registran el número de palieres que consigue completar.

- Velocidad sin balón sobre 20 m.: Las jugadoras salen desde la posición inicial y recorren 20 metros a la máxima velocidad posible. Y lo mismo realizado en conducción de balón, para medir la técnica.

- Velocidad sin balón sobre $10 \mathrm{~m}$. con cambio de dirección (CODA): Las jugadoras salen de la posición inicial y recorren, primero, $5 \mathrm{~m}$. pero a la mitad realizan un giro de $90 \%$. Y lo mismo realizado en conducción de balón, para medir la técnica.

- Test de Capacidad de Cambio de Dirección (CODA): para ello se utiliza el Modified Agility Test (MAT). Las jugadoras realizan el recorrido de $20 \mathrm{~m}$. en total. Salen de la posición inicial parados, recorren $5 \mathrm{~m}$. hasta el medio, giro de $90^{\circ}$ a la izquierda, $2.5 \mathrm{~m}$., giro de $180^{\circ}$ y recorren $5 \mathrm{~m}$., otro giro de $180^{\circ}$ hasta la mitad y de nuevo giro de $90^{\circ}$ y volver al inicio. Básicamente realizar el recorrido en forma de "T". Lo realizan forma libre, acción similar a la que realizan los jugadores en el terreno de juego (Yanci, et al, 2014). Y lo mismo realizado en conducción de balón, para medir el regate específico.

\subsection{PROCEDIMIENTO}

Los datos fueron recogidos en las instalaciones del club deportivo, previa solicitud y autorización a los padres, entrenadores y responsables técnicos del club deportivo. Se les permitía faltar una vez nada más, como en estudios similares (Sánchez, et al., 2014). El espacio de juego utilizado de 3 contra 3 se tomaron las medidas de $30 \times 20 \mathrm{~m}$. y para el 4 contra $436 \times 24 \mathrm{~m}$., realizadas en otras investigaciones (Kelly \& Drust, 2009; Soto \& Pérez, 2014b). Se realizan un total de 14 sesiones de entrenamiento basada en los SSGs (40 minutos de intervención) y se analizan las variables técnicas y de condición física, con una duración de $90 \mathrm{~m}$. por sesión de entrenamiento. Todas ellas juegan un partido de competición a la semana, habitualmente los sábados.

Los objetivos y contenidos de las sesiones realizadas se distribuye de la siguiente forma: las seis primeras sesiones se juegan sin porteros ni porterías con el espacio más reducido de $30 \times 20 \mathrm{~m}$., las seis siguientes sesiones se trabajan con porterías pequeñas y las dos últimas sesiones se hacen con porteros y porterías grandes, todas ellas con un espacio de $36 \times 24 \mathrm{~m}$. De esta forma la progresión realizada es desde juegos de posesión de balón, siguiendo 
con juegos de posesión, progresión y finalización en porterías pequeñas para terminar con juegos de posesión, progresión y finalización en porterías grandes. En todas las sesiones se trabajan los contenidos técnicos propios del modelo de juego combinativo ofensivo: control, pase, conducción, regate y tiro principalmente y tácticamente: los apoyos, desmarques, ayudas permanentes y espacios libres. No existen limitaciones en los toques de los jugadores y se juegan con las reglas propias del fútbol, menos la aplicación del fuera de juego y que se permite sacar de banda con el pie para darle mayor velocidad al juego al disponer siempre balones alrededor del espacio.

Antes de la intervención se realizaron los test de condición físico - técnico específicos medidos por el mismo evaluador. Todas las medidas seguían el mismo proceso, calentamiento tradicional de $15 \mathrm{~m}$. y al acabar se realizaban los test. Para conseguir gran fiabilidad y evitar errores en las mediciones se les enseñó en el entrenamiento anterior la ejecución correcta y se les permitió que lo practicaran. Se respetó la Declaración de Helsinki en todos sus términos.

\subsection{ANÁLISIS ESTADÍSTICO}

Para el tratamiento estadístico de los datos se utilizó el programa estadístico SPSS Statistics 20.0. Se calcularon los descriptivos (media y desviación típica). Se comprobó la normalidad de la muestra por medio de la Saphiro-Wilk. Se analizaron los efectos del programa de entrenamiento comparando los datos obtenidos en el pre-test y el post-test a través de la prueba t Student para muestras relacionadas. Las diferencias entre los resultados se consideran significativas si $p \leq 0,05$ o muy significativas $p \leq 0,00$. Por último, se analizaron las correlaciones bivariadas.

\section{RESULTADOS}

El objetivo del estudio ha sido analizar cómo influyen los juegos reducidos o SSGs sobre la condición física, técnica y los cambios de dirección (CODA) en jugadoras alevines de fútbol femenino, con un programa de entrenamiento de 14 sesiones. Tras la intervención realizada, las jugadoras presentan mejores valores medios de condición física (tabla 2). Mejoran en todos los aspectos analizados: fuerza de piernas, resistencia aeróbica, velocidad con cambio de dirección y en CODA, sin embargo no mejoran en la velocidad sin balón. En la fuerza explosiva la mejora es de más de $2 \mathrm{~cm}$. de media, en el caso del Course Navette la mejora es de un palier, en el caso de la velocidad sin balón con giro tenemos que se rebaja el tiempo entre 0,03 y 0,02 seg., y por último en CODA la mejora es pequeña.

Tabla 2. Resultados medios de la Condición Física pretest y postest.

\begin{tabular}{lcc}
\hline & Pretest & Postest \\
\hline Salto a Píes Juntos & 167,42 & 169,83 \\
Course Navette & 6,75 & 7,58 \\
Velocidad 10 m. con giro 90ํdecha & 2,64 & 2,32 \\
Velocidad 10 m. con giro 90 izquierda & 2,53 & 2,33 \\
Velocidad 20 m. & 3,53 & 3,83 \\
CODA (MAT) & 7,33 & 7,24 \\
\hline
\end{tabular}


En cuanto a los resultados técnicos, mejoran todos los resultados (tabla 3). Especialmente relevante es la mejora que han tenido en la conducción de balón sobre $10 \mathrm{~m}$., en concreto el giro hacía la derecha mejora sustancialmente sobre el giro hacía la izquierda, derecha 0,07 e izquierda 0,04 seg., respectivamente. También mejoran los resultados en la conducción lineal $(0,02$ seg.), si bien la mejora es inferior a la realizada en los $10 \mathrm{~m}$., y por último, también se mejora el regate - CODA (0,65 seg.).

Tabla 3. Resultados medios de técnica pretest y postest.

\begin{tabular}{lcc}
\hline & Pretest & Postest \\
\hline Conducción de balón $10 \mathrm{~m}$. con giro 90 a la derecha & 3,84 & 3,11 \\
Conducción de balón $10 \mathrm{~m}$. con giro 90 a la izquierda & 3,5 & 3,15 \\
Conducción de balón $20 \mathrm{~m}$. & 4,28 & 4,10 \\
Regate - CODA (MAT) con balón. & 10,15 & 9,50 \\
\hline
\end{tabular}

Existen diferencias estadísticamente muy significativas $(p \leq 0,00)$ en todas las variables analizadas, menos en el caso de la fuerza explosiva de salto a pies juntos, donde no existen diferencias significativas después de la intervención realizada (tabla 4), aunque sí que presenta mejores resultados.

Tabla 4. Diferencias significativas: Condición física.

\begin{tabular}{lccc}
\hline & Pretest & Postest & Sig. \\
\hline Salto a Píes Juntos & 167,42 & 169,83 & 0,183 \\
Course Navette & 6,75 & 7,58 & $0,000^{* *}$ \\
Velocidad $10 \mathrm{~m}$. derecha sin balón & 2,64 & 2,32 & $0,00^{* *}$ \\
Velocidad $10 \mathrm{~m}$. izquierda sin balón & 2,53 & 2,33 & $0,008^{* *}$ \\
Velocidad $20 \mathrm{~m}$. sin balón & 3,53 & 3,83 & $0,000^{* *}$ \\
CODA sin balón & 7,33 & 7,24 & $0,002^{* *}$ \\
\hline
\end{tabular}

${ }^{\star} p \leq 0,05 /{ }^{* \star} p \leq 0,00$

En cuanto a la técnica existen diferencias significativas en todas las variables analizadas (tabla 5). Ahora bien, en el caso de la conducción de balón sobre $20 \mathrm{~m}$. lineal la diferencia es significativa, mientras que en resto de variables analizadas las diferencias son muy significativas $(p \leq 0,00)$.

Tabla 5. Diferencias significativas: Técnica.

\begin{tabular}{lccc}
\hline & Pretest & Postest & Sig. \\
\hline Conducción de balón $10 \mathrm{~m}$. con giro $90^{\circ}$ a la derecha & 3,84 & 3,11 & $0,004^{* *}$ \\
Conducción de balón $10 \mathrm{~m}$. con giro $90^{\circ}$ a la izquierda & 3,5 & 3,15 & $0,000^{* *}$ \\
Conducción de balón $20 \mathrm{~m}$. & 4,28 & 4,10 & $0,024^{*}$ \\
Regate - CODA (MAT) con balón. & 10,15 & 9,50 & $0,007^{* *}$ \\
\hline
\end{tabular}

${ }^{*} p \leq 0,05 /{ }^{* *} p \leq 0,00$

En cuanto a las distintas correlaciones realizadas (tabla 6) tenemos que:

- La fuerza de salto se correlaciona positivamente, y de forma exclusiva, con la fuerza de salto postest. Del mismo modo que sucede con la resistencia aeróbica (Course Navette) que sólo tienen efectos positivos entre ellas. Al mejorar una se mejora la otra. 
- La velocidad sobre $10 \mathrm{~m}$. con giro $90^{\circ}$ a la derecha se correlaciona positivamente con el CODA sin balón tanto en ambos test.

- La Velocidad $10 \mathrm{~m}$. con giro 90 izquierda sin balón Postest se correlaciona positivamente con la velocidad sobre $20 \mathrm{~m}$. sin balón postest y una correlación altamente significativa con la mejora del regate-CODA con balón.

- La Velocidad $20 \mathrm{~m}$. sin balón se correlaciona muy significativamente con la conducción de balón sobre $20 \mathrm{~m}$. tanto en ambos test.

- La Velocidad $20 \mathrm{~m}$. sin balón Postest se correlaciona positivamente con la velocidad $10 \mathrm{~m}$. sin balón con giro $90^{\circ}$ postest.

- Los cambios de dirección (CODA) se correlaciona muy significativamente con la velocidad sobre $10 \mathrm{~m}$. con giro de $90^{\circ}$ a la derecha y con el CODA postest, sin balón en todos los casos.

- Los cambios de dirección (CODA) postest se correlaciona muy significativamente con la velocidad sobre $10 \mathrm{~m}$. con giro a la derecha sin balón y con los CODA sin balón pretest. También se relaciona significativamente con la velocidad sobre $10 \mathrm{~m}$. con giro a la derecha sin balón.

- La conducción sobre $10 \mathrm{~m}$. con giro a la derecha se correlaciona muy significativamente con la conducción sobre $20 \mathrm{~m}$.

- La conducción de balón $10 \mathrm{~m}$. con giro 90ํa la derecha Postest se correlaciona significativamente con la mejora del regate - CODA con balón.

- La conducción de balón $10 \mathrm{~m}$. con giro 90ํa la derecha Postest se correlaciona significativamente con el regate - CODA con balón.

- La conducción de balón $10 \mathrm{~m}$. con giro $90^{\circ}$ a la izquierda, se correlaciona significativamente con la conducción de balón sobre 10 $\mathrm{m}$. con giro a la derecha en el postest, así como con la conducción de balón sobre $20 \mathrm{~m}$. y con el regate - CODA con balón postest. Mientras que tiene una alta significación con la conducción de balón $20 \mathrm{~m}$. postest.

- La conducción de balón $10 \mathrm{~m}$. con giro 90 a la izquierda Postest presenta una correlación positiva con la conducción de balón $10 \mathrm{~m}$. con giro a la derecha postest y con la conducción sobre $10 \mathrm{~m}$. con la izquierda, así como con el regate - CODA con balón postest. Ahora bien, tiene una alta correlación significativa con la conducción de balón sobre $20 \mathrm{~m}$. postest. 
- La conducción de balón sobre $20 \mathrm{~m}$. se correlaciona significativamente con la conducción de balón sobre $10 \mathrm{~m}$. con giro a la izquierda y con el regate-CODA con balón postest. Mientras que se correlaciona muy significativamente con la conducción de balón $10 \mathrm{~m}$. con giro a la derecha, con la velocidad sin balón $20 \mathrm{~m}$. y con la conducción de balón $20 \mathrm{~m}$. postest.

- La conducción de balón $20 \mathrm{~m}$. Postest se relaciona significativamente con el regate - CODA postest, mientras que es muy significativa con: la conducción de balón sobre $10 \mathrm{~m}$. con giro a la izquierda pretest y postest, la velocidad sobre $20 \mathrm{~m}$. sin balón y con la conducción de balón $20 \mathrm{~m}$.

- Por último, el regate - CODA con balón postest se correlaciona muy significativamente con la velocidad sobre $10 \mathrm{~m}$. con giro a la izquierda, mientras que se relaciona significativamente con la conducción de balón sobre $10 \mathrm{~m}$. con giro a la derecha postest, con giro a la izquierda pretest, con la conducción de balón sobre $20 \mathrm{~m}$. pretest y postest y con el regate - CODA.

Tabla 6. Correlaciones por factores.

\begin{tabular}{|c|c|c|c|}
\hline \multirow[b]{2}{*}{ Salto a pies juntos } & \multirow[b]{2}{*}{ Salto a pies juntos Postest } & \multicolumn{2}{|c|}{$\begin{array}{l}\text { CorrelaciónSig. } \\
\text { de Pearson(bilateral) }\end{array}$} \\
\hline & & $0,951^{* *}$ & 0,000 \\
\hline Course Navette & Course Navette Postest & $0,959^{* *}$ & 0,000 \\
\hline \multirow{2}{*}{$\begin{array}{l}\text { Velocidad } 10 \mathrm{~m} \text {. con giro } 90^{\circ} \\
\text { derecha sin balón }\end{array}$} & CODA sin balón & $0,777^{\star \star}$ & 0,003 \\
\hline & CODA sin balón Postest & $0,727^{\star \star}$ & 0,007 \\
\hline \multirow[b]{2}{*}{$\begin{array}{l}\text { Velocidad } 10 \mathrm{~m} \text {. con giro } 90^{\circ} \\
\text { izquierda sin balón Postest }\end{array}$} & Velocidad 20 m. sin balón Postest & $0,662^{*}$ & 0,019 \\
\hline & $\begin{array}{l}\text { Regate - CODA (MAT) con balón } \\
\text { Postest }\end{array}$ & $0,711^{* *}$ & 0,010 \\
\hline \multirow{2}{*}{ Velocidad 20 m. sin balón } & Conducción de balón 20 m. & $0,881^{* *}$ & 0,000 \\
\hline & Conducción de balón 20 m. Postest & $0,809^{\star \star}$ & 0,001 \\
\hline Velocidad 20 m. sin balón Postest & $\begin{array}{l}\text { Velocidad } 10 \mathrm{~m} \text {. con giro } 90 \\
\text { izquierda sin balón Postest }\end{array}$ & $0,662^{*}$ & 0,019 \\
\hline \multirow[t]{2}{*}{ CODA sin balón } & $\begin{array}{l}\text { Velocidad } 10 \mathrm{~m} \text {. con giro 90ㅇ } \\
\text { derecha sin balón }\end{array}$ & $0,777^{* *}$ & 0,003 \\
\hline & CODA sin balón Postest & $0,973^{* *}$ & 0,000 \\
\hline \multirow{3}{*}{ CODA sin balón Postest } & $\begin{array}{l}\text { Velocidad } 10 \mathrm{~m} \text {. con giro } 90^{\circ} \\
\text { derecha sin balón Postest }\end{array}$ & $0,727^{* *}$ & 0,007 \\
\hline & $\begin{array}{l}\text { Velocidad } 10 \text { m. con giro } 90^{\circ} \\
\text { derecha sin balón }\end{array}$ & $0,616^{*}$ & 0,033 \\
\hline & CODA sin balón & $0,973^{* *}$ & 0,000 \\
\hline $\begin{array}{l}\text { Conducción de balón } 10 \mathrm{~m} . \text { con } \\
\text { giro } 90^{\circ} \text { a la derecha }\end{array}$ & Conducción de balón 20 m. & $0,752^{* \star}$ & 0,005 \\
\hline \multirow[t]{2}{*}{$\begin{array}{l}\text { Conducción de balón } 10 \mathrm{~m} \text {. con } \\
\text { giro } 90^{\circ} \text { a la derecha Postest }\end{array}$} & $\begin{array}{l}\text { Regate - CODA (MAT) con balón } \\
\text { Postest }\end{array}$ & $0,646^{*}$ & 0,023 \\
\hline & $\begin{array}{l}\text { Conducción de balón } 10 \mathrm{~m} \text {. con } \\
\text { giro } 90^{\circ} \text { a la izquierda Postest }\end{array}$ & $0,693^{*}$ & 0,012 \\
\hline \multirow{3}{*}{$\begin{array}{l}\text { Conducción de balón } 10 \mathrm{~m} \text {. con } \\
\text { giro } 90^{\circ} \text { a la izquierda }\end{array}$} & Conducción de balón 20 m. & $0,644^{*}$ & 0,024 \\
\hline & Conducción de balón $20 \mathrm{~m}$. Postest & $0,792^{* *}$ & 0,002 \\
\hline & $\begin{array}{l}\text { Regate - CODA (MAT) con balón } \\
\text { Postest }\end{array}$ & $0,669^{*}$ & 0,017 \\
\hline
\end{tabular}




\begin{tabular}{|c|c|c|c|}
\hline \multirow{4}{*}{$\begin{array}{l}\text { Conducción de balón } 10 \mathrm{~m} \text {. con } \\
\text { giro } 90^{\circ} \text { a la izquierda Postest }\end{array}$} & $\begin{array}{l}\text { Conducción de balón } 10 \mathrm{~m} \text {. con } \\
\text { giro } 90^{\circ} \text { a la derecha Postest }\end{array}$ & $0,686^{*}$ & 0,014 \\
\hline & $\begin{array}{l}\text { Conducción de balón } 10 \mathrm{~m} \text {. con } \\
\text { giro } 90^{\circ} \text { a la izquierda }\end{array}$ & $0,693^{*}$ & 0,012 \\
\hline & Conducción de balón 20 m. Postest & $0,708^{* *}$ & 0,010 \\
\hline & $\begin{array}{l}\text { Regate - CODA (MAT) con balón } \\
\text { Postest }\end{array}$ & $0,695^{*}$ & 0,012 \\
\hline \multirow{5}{*}{ Conducción de balón 20 m. } & $\begin{array}{l}\text { Conducción de balón } 10 \mathrm{~m} \text {. con } \\
\text { giro } 90^{\circ} \text { a la derecha }\end{array}$ & $0,752^{\star \star}$ & 0,005 \\
\hline & $\begin{array}{l}\text { Conducción de balón } 10 \mathrm{~m} \text {. con } \\
\text { giro } 90^{\circ} \text { a la izquierda }\end{array}$ & $0,644^{*}$ & 0,024 \\
\hline & Velocidad 20 m. sin balón & $0,881^{\star *}$ & 0,000 \\
\hline & Conducción de balón $20 \mathrm{~m}$. Postest & $0,855^{\star *}$ & 0,000 \\
\hline & $\begin{array}{l}\text { Regate - CODA (MAT) con balón } \\
\text { Postest }\end{array}$ & $0,602^{*}$ & 0,038 \\
\hline \multirow{5}{*}{ Conducción de balón 20 m. Postest } & $\begin{array}{l}\text { Conducción de balón } 10 \mathrm{~m} \text {. con } \\
\text { giro } 90^{\circ} \text { a la izquierda }\end{array}$ & $0,792^{\star *}$ & 0,002 \\
\hline & $\begin{array}{l}\text { Conducción de balón } 10 \mathrm{~m} \text {. con } \\
\text { giro } 90^{\circ} \text { a la izquierda Postest }\end{array}$ & $0,708^{* *}$ & 0,010 \\
\hline & Velocidad 20 m. sin balón & $0,809^{* *}$ & 0,001 \\
\hline & Conducción de balón 20 m. & $0,855^{\star *}$ & 0,000 \\
\hline & $\begin{array}{l}\text { Regate - CODA (MAT) con balón } \\
\text { Postest }\end{array}$ & $0,630^{*}$ & 0,028 \\
\hline \multirow{7}{*}{$\begin{array}{l}\text { Regate - CODA (MAT) con balón } \\
\text { Postest }\end{array}$} & $\begin{array}{l}\text { Velocidad } 10 \mathrm{~m} \text {. izquierda sin balón } \\
\text { Postest }\end{array}$ & $0,711^{\star *}$ & 0,010 \\
\hline & $\begin{array}{l}\text { Conducción de balón } 10 \mathrm{~m} \text {. con } \\
\text { giro } 90^{\circ} \text { a la derecha Postest }\end{array}$ & $0,646^{*}$ & 0,023 \\
\hline & $\begin{array}{l}\text { Conducción de balón } 10 \mathrm{~m} \text {. con } \\
\text { giro } 90^{\circ} \text { a la Izquierda }\end{array}$ & $0,669^{*}$ & 0,017 \\
\hline & $\begin{array}{l}\text { Conducción de balón } 10 \mathrm{~m} \text {. con } \\
\text { giro } 90^{\circ} \text { a la Izquierda Postest }\end{array}$ & $0,695^{\star}$ & 0,012 \\
\hline & Conducción de balón 20 m. & $0,602^{*}$ & 0,038 \\
\hline & Conducción de balón 20 m. Postest & $0,630^{*}$ & 0,028 \\
\hline & Regate - CODA (MAT) con balón & $0,696^{*}$ & 0,012 \\
\hline
\end{tabular}

${ }^{* *}$. La correlación es significativa al nivel 0,01 (bilateral).

*. La correlación es significante al nivel 0,05 (bilateral).

\section{DISCUSIÓN}

La investigación tiene por objetivo analizar los efectos que tienen los juegos reducidos específicos en fútbol sobre la condición física y técnica de una muestra de jugadoras de fútbol, utilizando para ello un programa de entrenamiento con 14 sesiones con tareas jugadas en situaciones de tres contra tres y cuatro contra cuatro.

Los resultados muestran que existe una mejora de todas las variables analizadas, a excepción de la velocidad sobre $20 \mathrm{~m}$. sin balón. Por lo tanto, de forma general se mejora la fuerza de piernas, la resistencia aeróbica, la agilidad y la velocidad con cambios de dirección con y sin balón. Estos datos coinciden con los extraídos por Sánchez et al. (2014), eso sí en población masculina, por lo tanto se produce el mismo efecto en la resistencia aeróbica, la agilidad y la velocidad con indiferencia del sexo de los sujetos con esta edad. También 
coincide con los estudios de Craig et al. (2014) y de Halouani, Chtourou, Dellal, Chaouachi y Chamari (2014) sobre la mejorar de la resistencia aeróbica. Sin embargo no coinciden con los resultados de Sánchez, et al. (2014) en la fuerza piernas ya que en nuestro estudio sí que presentan mejora en los valores medios. Si bien, coincidimos con el estudio de Sánchez et al. (2014) en cuanto a las diferencias significativas tras la intervención y aquí sí que existe coincidencia en la resistencia aeróbica y la agilidad (CODA).

En cuanto a las variables técnicas en este trabajo coinciden con los realizados con hombres por Pérez, et al (2012), Sánchez et al. (2014) y Soto y Pérez (2014b) en la mejora de la conducción y del regate. tanto por su mejora de los valores medios como en la existencia de diferencias significativas.

Al igual que otros estudios la utilización de los SSGs es adecuada, útil, efectiva y contextualizada a la realidad de juego y la competición (Craig, Andrew, Nicholas \& Taisuke, 2014; Halouani et al., 2014; Pérez, et al., 2012; Sánchez, et al., 2016; Wein, 1995), siendo una alternativa a los entrenamientos más tradicionales y dedicados al entrenamiento separado sin tener en cuenta la realidad del juego (Casamichana et. al. 2015; Rampinini, et al., 2007; Sánchez, et al., 2016), y por supuesto, transferibles a las situaciones específicas de competición (Casamichana et. al. 2015).

De nuevo los datos coinciden con los que expresan Febré, et al. (2015), Katis y Kellis (2009) y Sánchez et al., (2014) que el objetivo de los programas de entrenamiento con jóvenes tienen que estar orientados a la mejora de la condición técnica y física, en población de mujeres pre púberes. Así como que coinciden con los estudios de Craig et al. (2014) y Sánchez, et al. (2014) cuando señalan la importancia que tiene este tipo de entrenamientos son adecuados para deportistas jóvenes, ya que en la mayoría de las publicaciones científicas se centran en la población adulta. Pueden ayudar a preparar jugadores con mejores capacidades de pensamiento y toma de decisiones en diferentes situaciones (Cristian, et al., 2015; Martínez, et. al., 2015; Young \& Rogers, 2104).

Este trabajo no se relaciona con el realizado por Castillo, Fernández, Cinchilla y Álvarez (2012) que muestran correlación entre la fuerza de salto vertical y el CODA, aspecto este que en nuestro estudio no tiene dicha correlación como en el estudio de Pardeiro y Yanci (2017), si bien es cierto que su investigación se centra en jugadores profesionales y semi profesionales respectivamente, y en nuestro caso es en jugadores en formación y con niveles físicos de fuerza diferente, ya que todavía no han desarrollado toda su fuerza. Del mismo modo se relaciona con los resultados objetivos en el estudio de Yanci et al (2013) y Yanci, et al. (2014), porque se relaciona la velocidad sobre espacios cortos con la agilidad MAT o CODA, así como con el estudio de Arin, Jansson y Skarphagen (2012) que muestran una relación significativa, con valores de significación similar, entre la velocidad sobre 10 y $20 \mathrm{~m}$. con el test CODA, eso sí la muestra son totalmente diferente. Por último, se relaciona con el estudio de Yanci, et al. (2016) en una muestra similar de edad, pero en un deporte totalmente distinto, como es el atletismo. Por último, se relaciona con el estudio realizado por Young y Rogers (2014), al determinar que los juegos reducidos mejoran el rendimiento de la agilidad. 
Nuestro estudio se relaciona con los datos obtenidos por Yanci et al (2013), en que la alta variabilidad produce mejoras en los cambios de dirección (CODA) y la velocidad lineal, como también sucede en nuestro caso ya que se mejora de forma significativa los cambios de dirección y la velocidad línea con la aplicación del programa de entrenamiento basado en SSGs.

No se puede relacionar la investigación realizada con otras con una población objeto de estudio similar, y que demuestra que la mejora de la velocidad y la conducción sobre $10 \mathrm{~m}$. con giro de $90^{\circ}$ se relaciona de forma significativa con la mejora de los cambios de dirección - CODA con y sin balón, aspectos estos claves en el juego del fútbol como señalan varios estudios (Bangsbo, et al., 2006; Yanci, et al., 2014).

Por último, sería muy interesante llevar a cabo más estudios donde se analicen estos resultados en comparación con distintas categorías de fútbol femenino exclusivamente, y por supuesto, que en comparación con población masculina, tanto con programas de entrenamiento basado en SSGs como con otro tipo de programas de entrenamiento.

\section{CONCLUSIONES}

Los resultados extraídos muestran que se ha mejorado la condición física, la técnica y los CODA de las jugadoras de fútbol a través de un programa de entrenamiento en SSGs. Se mejora la resistencia aeróbica, la velocidad, la agilidad, la técnica de conducción de balón y de cambios de dirección (CODA) todo ello dentro de un entorno de especificad del entrenamiento siendo todos ellos condiciones y cualidades necesarias para jugar al fútbol, sin embargo no se mejora la fuerza explosiva de forma significativa, en una muestra de jugadoras de fútbol pre púberes.

La mejora de velocidad lineal, la velocidad y la conducción de balón con cambios de dirección mejoran bastante los cambios de dirección - CODA y el regate- CODA, ambos aspectos específicos y altamente relacionados con el rendimiento en el fútbol, por ello el entrenamiento de estos aspectos es muy recomendado en estas edades para provocar un mejor rendimiento actual y futuro en el fútbol.

Por lo tanto la realización de un programa de entrenamiento compuesto por 14 sesiones en las que se incluyen juegos reducidos o SSGs de fútbol, puede ser usado con futbolistas jóvenes para mejorar algunas cualidades físicas, los cambios de dirección - CODA y aspectos técnicos propios y específicos del fútbol, como la conducción y el regate. Siendo los aprendizajes adquiridos transferibles a situaciones reales de juego. De esta forma podemos considerar que son un método adecuado y muy ventajoso para el entrenamiento en las etapas iniciales o de formación en jugadoras de fútbol. 


\section{REFERENCIAS BIBLIOGRAFÍCAS}

Abrantes, C. I., Nunes, M. I., Maçãs, V. M., Leite, N. M. \& Sampaio, J. E. (2012). Effects of the number of players and game type constraints on heart rate, rating of perceived exertion, and technical actions of small-sided soccer games. The Journal of Strength \& Conditioning Research, 26(4), 976-981. https://doi.org/10.1519/JSC.0b013e31822dd398

Arin, A., Jansson, D. \& Skarphagen, K. (2012). Maximal unilateral leg strength correlates with linear sprint and change of direction speed. Tesis Doctoral. University of Göteborg. Göteborg.

Ayala, F., de Baranda, P. S., Cejudo, A. \& De Ste Croix, M. (2010). Efecto de un programa de estiramientos activos en jugadoras de fútbol sala de alto rendimiento. Cultura, Ciencia y Deporte, 5(15), 159-167. https://doi.org/10.12800/ccd.v5i15.105

Bangsbo, J., Mohr, M. \& Krustrup, P. (2006). Physical and metabolic demands of training and match-play in the élite football player. Journal of Sports Science, 24, 665-674. https://doi.org/10.1080/02640410500482529

Barbero-Álvarez, J. C., Barbero-Álvarez, V., Gómez, M., \& Castagna, C. (2009). Análisis cinemático del perfil de actividad en jugadoras infantiles de fútbol mediante tecnología GPS. Kronos, 8(15), 35-42.

Casamichana, D., Castellano, J., González-Morán, A., García-Cueto, H. \& García-López, J. (2011). Demanda fisiológica en juegos reducidos de fútbol con diferente orientación del espacio. RICYDE. Revista Internacional De $\begin{array}{llll}\text { Ciencias Del } & \text { 141-154. }\end{array}$ https://doi.org/10.5232/ricyde2011.02306

Casamichana, D., San Román, J., Calleja, J. \& Castellano, J. (2015). Los juegos reducidos en el entrenamiento del fútbol. Futbol de libro S.L. Editorial.

Castellano, J., Casamichana, D. \& Dellal, A. (2013). Influence of game format and number of players on heart rate responses and physical demands in small-sided soccer games. Journal of Strength and Conditioning Journal, 27(5), 1295-1303. https://doi.org/10.1519/JSC.0b013e318267a5d1

Castillo, A., Fernández, J. C., Chinchilla, J. L. \& Álvarez, E. (2012). Relationship between muscular strength and sprints with changes of direction. Journal of Strength and Conditioning Research, 26(3), 725-732. https://doi.org/10.1519/JSC.0b013e31822602db

Cook, T.D. \& Reichardt, CH. S. (1986). Métodos cualitativos y cuantitativos en investigación evaluativa. Madrid: Morata.

Craig B.H, Andrew E. K., Nicholas D.G. \& Taisuke, K. (2014). Small- sided games for young athletes: is game specificity influential? Journal of Sports Sciences, 32(4), 336-344. https://doi.org/10.1080/02640414.2013.824600

Cristian, L., Riller, R., René, B., Denise, M., Milton, M. \& Alcides, S. (2015). Technical and tactical soccer players' performance in conceptual small-sided games. Motriz: Revista De Educação Física, 21(3), 312-320. https://doi.org/10.1590/S1980-65742015000300013

Del Río, J., Flores, P. J., Bautista, J., Barajas, L. T., Tamara, R. \& Gómez, E. (2015). Efectos de un programa de flexibilidad en el desarrollo de la fuerza muscular en jugadoras de futbol femenil. Educación Física y Ciencia, 17(2), $1-9$. 
Dellal, A., Drust, B. \& Lago-Penas, C. (2012). Variation of activity demands in small-sided soccer games. International Journal of Sports Medicine, 33(05), 370-375. https://doi.org/10.1055/s-0031-1295476

Dellal, A., Hill-Haas, S., Lago-Penas, C. \& Chamari, K. (2011). Small-sided games in soccer: amateur vs. professional players's physiological responses, physical, and technical activities. Journal of Strength \& Conditioning Research, 25(9), 2371-2381.https://doi.org/10.1519/JSC.0b013e3181fb4296

Febré, R., Javier, L., Casamichana, D., Chirosa, I., Martín-Tamayo, I. \& Pablos, C. (2015). Influencia de la densidad de jugadores sobre la frecuencia cardíaca y respuestas técnicas en jóvenes jugadores de fútbol. RICYDE. Revista Internacional De Ciencias Del Deporte, 11(40), 116-128. https://doi.org/10.5232/ricyde2015.04002

Fradua, L., Zubillaga, A., Caro, Ó., Fernández-García, Á. I., Ruiz-Ruiz, C. \& Tenga, A. (2013). Designing small-sided games for training tactical aspects in soccer: Extrapolating pitch sizes from full-size professional matches. Journal of sports sciences, 31(6), 573-581. https://doi.org/10.1080/02640414.2012.746722

Gonçalves, B.V., Figueira, B.E., Maças, V. \& Sampaio, J. (2014). Effect of player position on movement behaviour, physical and physiological performances during an 11-a-side football game. Journal of Sports Sciences, 32(2):191-199. https://doi.org/10.1080/02640414.2013.816761

González-Víllora, S., García-López, L.M. \& Contreras-Jordán, O.R. (2015). Evolución de la toma de decisiones y la habilidad técnica en fútbol / Decision Making and Skill Development In Youth Football Players. Revista Internacional de Medicina y Ciencias de la Actividad Física y el Deporte, 15(59), 467-487. http://dx.doi.org/10.15366/rimcafd2015.59.005

González, I., Sánchez, D., Amado, D., Pulido, J. J., López, J. M. \& Leo, D. M. (2013). Análisis de la cohesión, la eficacia colectiva y el rendimiento en equipos femeninos de fútbol. Apunts: Educació Física I Esports, (114), 65-71. https://doi.org/10.5672/apunts.2014-0983.es.(2013/4).114.07

Hernández, J. (1994). Fundamentos del deporte: análisis de la estructura de los juegos deportivos. Barcelona: Ed. Inde.

Hill-Haas, S. V., Dawson, B., Impellizzeri, F. M. \& Coutts, A. J. (2011). Physiology of small-sided games training in football: a systematic review. Sports medicine, 41(3), 199-220. https://doi.org/10.2165/11539740-000000000$\underline{00000}$

Halouani, J., Chtourou, H., Dellal, A., Chaouachi, A., \& Chamari, K. (2014). Physiological responses according to rules changes during 3 vs. 3 small-sided games in youth soccer players: stop-ball vs. small-goals rules. Journal Of Sports Sciences, 32(15), 1485-1490. https://doi.org/10.1080/02640414.2014.899707

Katis, A. \& Kellis, E. (2009). Effects of small-sided games on physical conditioning and performance in young soccer players. Journal of sports science and medicine, 8, 374-380.

Kelly, D.M. \& Drust, B. (2009). The effect of pitch dimensions on heart rate responses and technical demands of small-sided soccer games in elite players. Journal of Science and Medicine in Sport, 12(4), 475-479. https://doi.org/10.1016/j.jsams.2008.01.010 
Little, T. (2009). Optimizing the use of soccer drills for physiological development. Strength and Conditioning Journal, 31(3), 67-74. https://doi.org/10.1519/SSC.0b013e3181a5910d

Little, T., \& Williams, A. G. (2006). Suitability of soccer training drills for endurance training. The Journal of Strength \& Conditioning Research, 20(2), 316-319. https://doi.org/10.1519/00124278-200605000-00014

Martone, D., Giacobbe, M., Capobianco, A., Imperlini, E., Mancini, A., Capasso, M., Buono, P \& Orrù, S. (2017). Exercise intensity and technical demands of small-sided soccer games for under-12 and under-14 players: effect of area per player. Journal of Strength and Conditioning Research, 31(6), 1486-1492. https://doi.org/10.1519/JSC.0000000000001615

Martínez, I. M., Reigal, R. E., Chirosa, L. J., Hernández, A., Ríos, I. C., Tamayo, I. M. \& Barrilao, R. G. (2015). Efectos de un programa de juegos reducidos en la percepción subjetiva del esfuerzo en una muestra de chicas adolescentes. Cuadernos De Psicología Del Deporte, 15(3), 89-98. https://doi.org/10.4321/S1578-84232015000300008

Nevado-Garrosa, F. \& Suárez-Arrones, L. (2015). Comparación de las demandas físicas de tareas de fútbol reducido y la competición en jugadoras de fútbol sub 13. Cultura, Ciencia y Deporte, 10(30), 235-243. https://doi.org/10.12800/ccd.v10i30.592

Owen, A. L., Wong, D. P., Paul, D. \& Dellal, A. (2012). Effects of a periodized small- sided game training intervention on physical performance in elite professional soccer. The Journal of Strength \& Conditioning Research, 26(10), 2748-2754. https://doi.org/10.1519/JSC.0b013e318242d2d1

Owen, A., Twist, C. \& Ford, P. (2004). Small-sided games: the physiological and technical effect of altering pitch size and player numbers. Insight F.A. Coaches Assoc. J, 7(2), 50-53.

Pardeiro, M. \& Yancy, J. (2017). Efectos del calentamiento en el rendimiento físico y en la percepción psicológica en jugadores semi profesionales de fútbol. RYCYDE. Revista Internacional de Ciencias del Deporte, 48(13), 104116. https://doi.org/10.5232/ricyde2017.04802

Parlebas, P. (2001). Juegos, deporte y sociedad. Léxico de praxiología motriz. Barcelona: Paidotribo.

Pérez, S. Sánchez, J. \& Urchaga, D. (2015). Los motivos para la participación en fútbol: Estudio por categorías y nivel de competición de los jugadores. Cultura, Ciencia y Deporte, 10, 187-198. https://doi.org/10.12800/ccd.v10i30.588

Pérez, S., Sánchez, J., Sánchez, I. \& Yagüe, J. Mª (2012). Comparación Del Grado De Intervención Técnico Ofensiva 3 Contra 3 Y El Futbol 7. VII Congreso Internacional de la Asociación Española de Ciencias del Deporte.

Pita, S. \& Pértega, S. (2002). Investigación: Investigación cuantitativa y cualitativa. Cad Aten Primaria, 9, 76-78.

Rampinini, E., Impellizzeri, F.M., Castagna, C., Abt, G., Chamari, K., Sassi, A. \& Marcora, S.M. (2007). Factors influencing physiological responses to smallsided soccer games. Journal of sports sciences, 25(6), 659-66. https://doi.org/10.1080/02640410600811858

Robles, J., Giménez, F. J. \& Abad, M. T. (2011). Metodología utilizada en la enseñanza de los contenidos deportivos durante la ESO. Revista 
Internacional de medicina y ciencias de la actividad física y el deporte, 11(41), 35-57.

Sánchez-Sánchez, J., Carretero, M., Assante, G., Casamichana, D. \& Arcos, A. L. (2016). Efectos del marcaje al hombre sobre la frecuencia cardíaca, el esfuerzo percibido y la demanda técnico-táctica en jóvenes jugadores de fútbol. RICYDE. Revista Internacional De Ciencias Del Deporte, 12(44), 94106. https://doi.org/10.5232/ricyde2016.04401

Sánchez, J., Yagüe, J.M., Fernández, R.C. \& Petisco, C. (2014). Efectos de un entrenamiento con juegos reducidos sobre la técnica y la condición física de jóvenes futbolistas. RICYDE. Revista internacional de ciencias del deporte, 37(10), 221-234. https://doi.org/10.5232/ricyde2014.03704

Santiago, A., Granados, C., Quintela, K. \& Yanci, J. (2015). Diferencias entre jugadores de fútbol de distintas edades en la capacidad de aceleración, cambio de dirección y salto. Cultura, Ciencia y Deporte, 10, 135-143. https://doi.org/10.12800/ccd.v10i29.551

Soto, F. \& Pérez, S. (2014a). Análisis de los tiempos de juego, posesión y duración de jugadas en SSGs y fútbol 11. En Villar, F. Claver, F. \& Fuentes, J. P. VIII Congreso Internacional de la Asociación Española de Ciencias. Cáceres: Asociación Española de Ciencias del Deporte.

Soto, F. \& Pérez, S. (2014b). Grado de intervención técnica en juegos reducidos de fútbol y fútbol 11 infantil. En Villar, F. Claver, F. \& Fuentes, J. P. VIII Congreso Internacional de la Asociación Española de Ciencias. Cáceres: Asociación Española de Ciencias del Deporte.

Vitoria, M. (2005). Motivación en deportistas juveniles de alta competición. Madrid: Editorial Gymnos.

Wein, H. (1995). Fútbol a la medida del niño. Madrid: Gymnos.

Yanci, J., Castillo, D., Vizcay, J.J., Pitillas, I. \& Iturricastillo, A. (2016). Relación entre la capacidad de aceleración, cambio de dirección y salto horizontal en atletas jóvenes. RICCAFD, Revista Iberoamericana de la Actividad Física y el Deporte, 5(2), 1-15.

Yanci, J., García, A., Castillo, D. \& Rivero, L.A. (2014). Evaluación y relación entre distintos parámetros de condición física en futbolistas semi profesionales. Retos. Nuevas tendencias en Educación Física, Deporte y Recreación, 26, 114-117.

Yanci, J., Reina, R., Los Arcos, A., \& Cámara, J. (2013). Effects of Different Contextual Interference Training Programs on Straight Sprinting and Agility Performance of Primary School Students. Journal Of Sports Science \& Medicine, 12(3), 601-607.

Young, W. \& Rogers, N. (2014). Effects of small-sided game and change-ofdirection training on reactive agility and change-of-direction speed. Journal of $\begin{array}{lll}\text { Sports } \quad \text { Sciences, 32:4, 307-314. } & \text { 32 }\end{array}$ https://doi.org/10.1080/02640414.2013.823230

Número de citas totales / Total referentes: 50 (100\%)

Número de citas propias de la revista / Journal's own referentes: 2 (4\%)

Rev.int.med.cienc.act.fís.deporte - vol. 19 - número 74 - ISSN: 1577-0354 